EUROPEAN JOURNAL OF PURE AND APPLIED MATHEMATICS

Vol. 15, No. 1, 2022, 238-248

ISSN 1307-5543 - ejpam.com

Published by New York Business Global

\title{
Investigation of the Infinite Discrete Levels from Finite discrete Levels in Position Dependent Mass Quantum Systems
}

\author{
Biswanath Rath ${ }^{1}$, Jihad Asad ${ }^{2, *}$, Hussein Shanak ${ }^{2}$, Rabab Jarrar ${ }^{2}$, \\ Mohammed K. A. Kaabar ${ }^{3}$ \\ ${ }^{1}$ Department of Physics, Maharaja Sriram Chandra Bhanj Deo University, Takatpur, \\ Baripada 757003, Odisha, India \\ ${ }^{2}$ Department of Physics,Faculty of Applied Sciences, Palestine Technical University-Kadoorie, \\ Tulkarm, Palestine \\ ${ }^{3}$ Institute of Mathematical Sciences, Faculty of Science, University of Malaya, Kuala Lumpur \\ 50603, Malaysia
}

\begin{abstract}
We find quantum systems having finite distinct discrete energy levels can reflect infinite distinct energy levels under suitable form of position dependent mass systems. A model example has been investigated considering the fractional Harmonic Oscillator [6].In addition to this, we also notice the same behaviour in other quantum models. In all the cases, we find finite distinct discrete levels becoming infinite distinct discrete levels under position dependent mass quantum systems without the change in the potential energy.
\end{abstract}

2020 Mathematics Subject Classifications: 35J10, 34L40, 11C20, 15B57

Key Words and Phrases: Infinite levels, Position dependent mass, Matrix diagonalisation method, Fractional potentials, Exponential potentials, von Roos model

\section{Introduction}

Bounded quantum structures are associated with actual strength levels [2] - These systems can have infinite or finite distinct discrete levels. A model infinite quantum system is $[6]$ :

$$
h=p^{2}+\lambda x^{2}+\frac{x^{2}}{\left(1+g x^{2}\right)}
$$

*Corresponding author.

DOI: https://doi.org/10.29020/nybg.ejpam.v15i1.4190

Email addresses: biswanathrath10@gmail.com (B. Rath), j.asad@ptuk.edu.ps (J. Asad), h.shanak@ptuk.edu.ps (H. Shanak), r.jarrar@ptuk.edu.ps (R. Jarrar),

mohammed.kaabar@wsu.edu (M. K. A. Kaabar) 
This systems reflect infinite levels as long as $\lambda \gg 0$. However a drastic change in energy levels is noticed in the limit $\lambda=0$. Under this condition model Hamiltonian becomes

$$
H=p^{2}+\frac{x^{2}}{\left(1+x^{2}\right)}
$$

The model potential satisfies the condition $V(x)=\frac{x^{2}}{\left(1+x^{2}\right)} \ll 1$, which is basically the reason for "distinct discrete levels"(DDL). This mannequin has been studied detail by Rath and Kaabar [11]. However, we consider here a slightly modified DDL as:

$$
H^{1}=p^{2}+\frac{x^{2}}{\left(1+2 x^{2}\right)}
$$

which is recast as

$$
=\left[p^{2}+M(x) x^{2}\right]
$$

where $M(x)=\frac{1}{\left(1+2 x^{2}\right)}$. Energy levels of this oscillator has been reflected in Fig. 1. Below, we focus our attention on new models without the change in potential energy as follows.

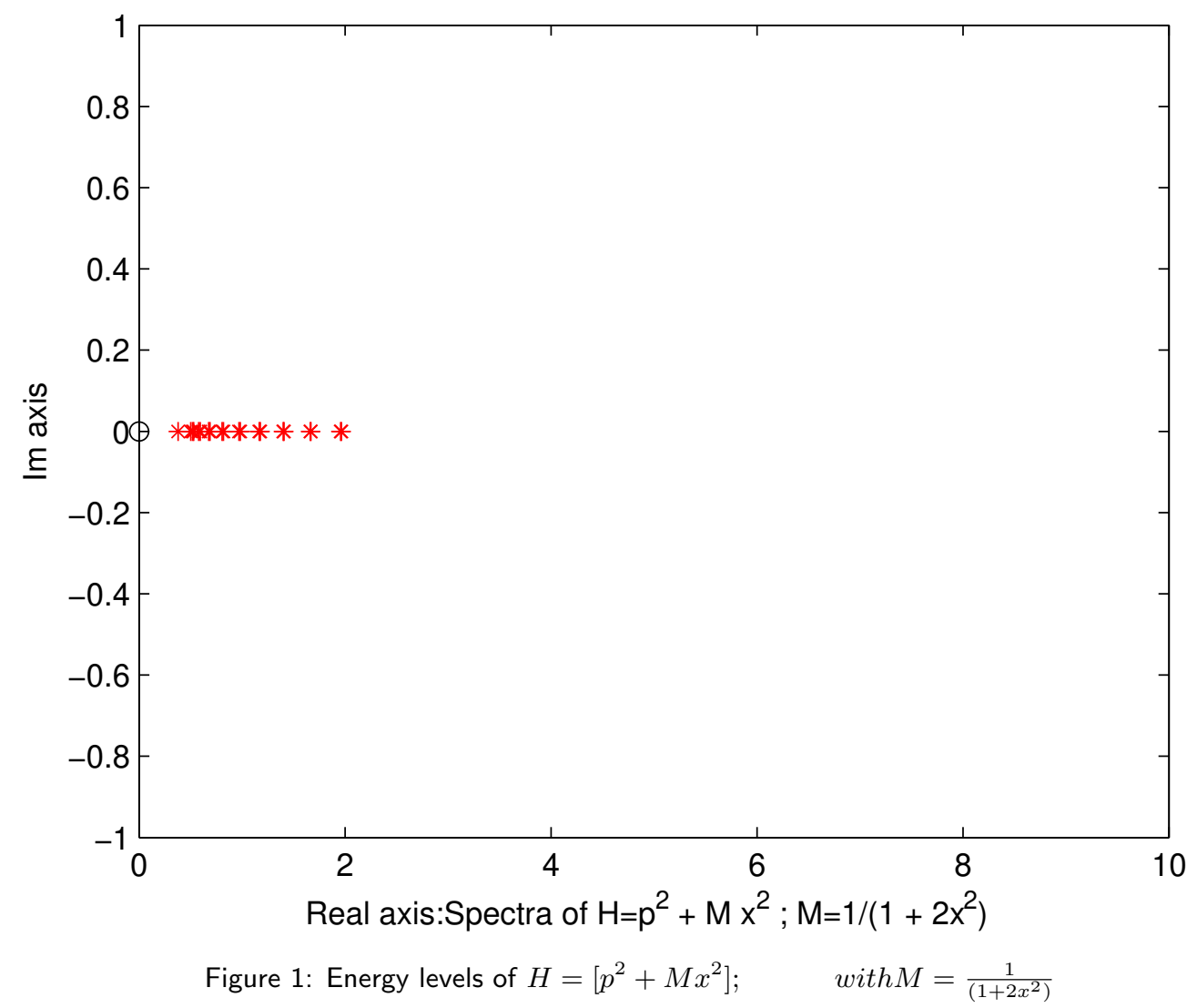




\section{Position dependent mass system without potential change}

At the outset, we would like to state that all our equations are valid subject to validity of commutation relation

$$
\begin{gathered}
{[x, p]=i} \\
H_{P D M}=p^{2} / M(x)+M(x) x^{2 N}=\frac{p^{2}}{M}+V(x)
\end{gathered}
$$

Hence, the corresponding $\mathrm{M}(\mathrm{x})$ can be written as

$$
M(x)=M=\frac{V(x)}{x^{2 N}} \quad N=1,2,3 \ldots
$$

and so on. In fact, for $\mathrm{N}=1$ model Hamiltonian was also previously proposed by Mathews and Lakshnman for the study of nonlinear analysis in view of its potential applications in quantum field theory [4]. As such the Hamiltonian is not self-adjoint $\left(H_{P D M} \neq H_{P D M}^{\dagger}\right)$ $[1,3,7,9,13-15]$. In its self adjoint form, it is expressed as [15]:

$$
H_{P D M S}=H^{(2)}=\left[-\frac{1}{M^{1 / 4}} \partial_{x} \frac{1}{M^{1 / 2}} \partial_{x} \frac{1}{M^{1 / 4}}+M(x) x^{2}\right]
$$

Hence the resultant Hamiltonian becomes von Roos model Hamiltonian [13, 14]. Energy levels of this Hamiltonian are reflected in Fig. 2.

In subsequent applications of von Roos model we use

$$
p^{2} / M=T=\left[-\frac{1}{M^{1 / 4}} \partial_{x} \frac{1}{M^{1 / 2}} \partial_{x} \frac{1}{M^{1 / 4}}\right]
$$

Hence in all the PDM calculations, the corresponding Hamiltonian is self-adjoint in nature $(T=$ $\left.T^{\dagger}\right)$. Apart from this we present a few models as

Case-I: Trivial exponential model

$$
\left.H^{(3)}=p^{2}+10\left(1-\exp \left(-x^{2}\right)\right)\right)
$$

Hence, the corresponding PDM operator can be written as

$$
H_{P D M}^{(4)}=H^{(4)}=\frac{p^{2}}{M}+10\left(1-\exp \left(-x^{2}\right)\right) ; \quad \text { with } M=10 \frac{\left[1-\exp \left(-x^{2}\right)\right]}{x^{2}}
$$

The respective energy levels are reflected in Fig. 3 and Fig. 4, respectively.

Similarly, we select a model PDM Hamiltonian as

$$
H^{(5)}=\frac{p^{2}}{M}+10\left(1-\exp \left(-x^{4}\right)\right) ; \quad \text { with } M=\frac{\left.10\left[1-\exp \left(-x^{4}\right)\right)\right]}{x^{4}}
$$

The respective energy levels are reflected in Fig. 5. One can also extend this approach to

$$
H^{(6)}=\frac{p^{2}}{M}+10\left(1-\exp \left(-x^{6}\right)\right) ; \quad \text { with } M=\frac{\left.10\left[1-\exp \left(-x^{6}\right)\right)\right]}{x^{6}}
$$




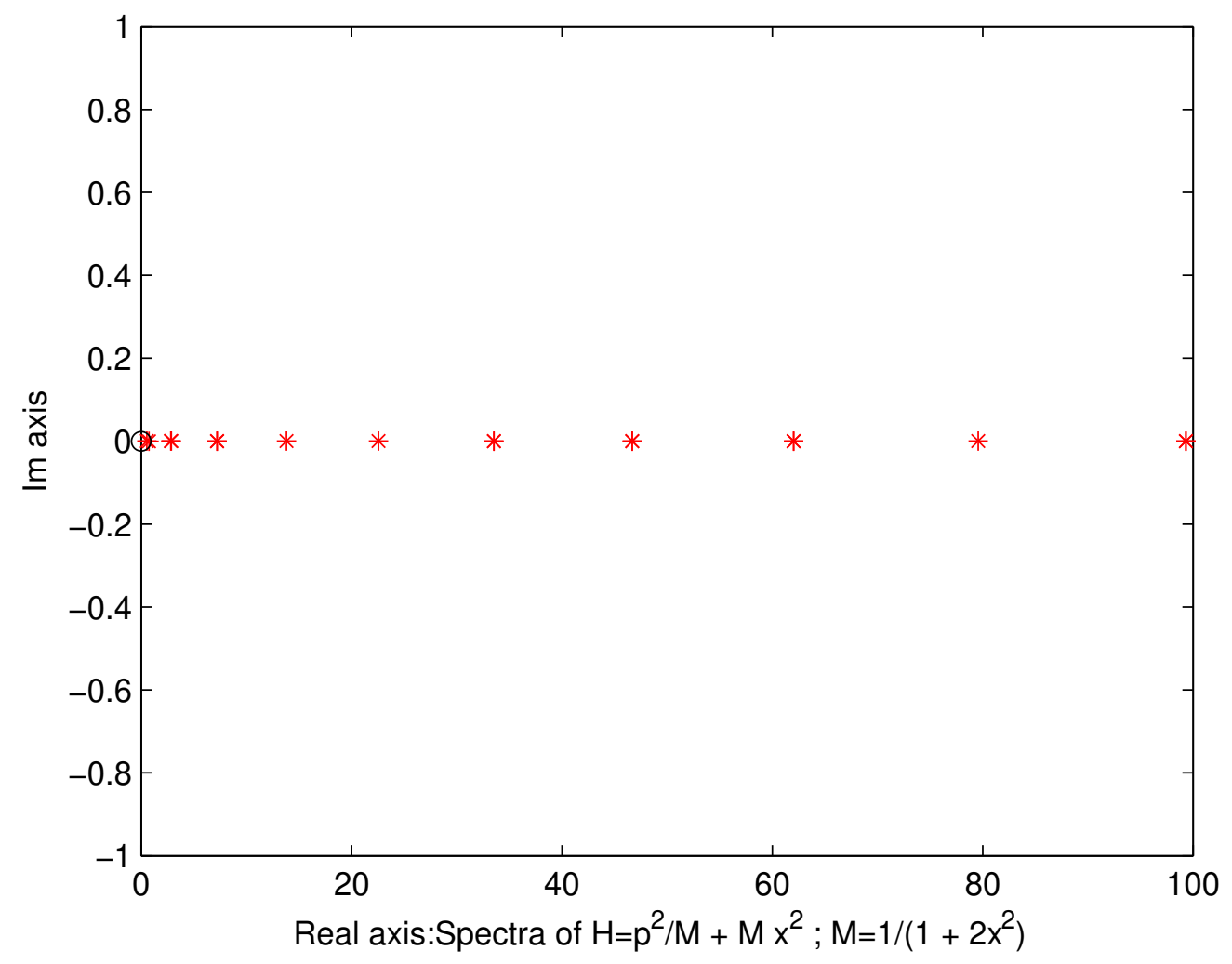

Figure 2: Energy levels of $H=\left[p^{2} / M+M x^{2}\right] ; \quad$ with $M=\frac{1}{\left(1+2 x^{2}\right)}$

The respective energy levels are reflected in Fig. 6.

\section{Case-II:Non-trivial exponential model}

Here, we consider the model Hamiltonian as

$$
H^{(7)}=p^{2}+\frac{\left(1-\exp \left(-x^{2}\right)\right)}{\left(1+\exp \left(-x^{2}\right)\right)}
$$

whose energy levels has finite distinct discrete levels as seen in Fig. 7.

The corresponding PDM operators as

$$
H^{(8)}=\frac{p^{2}}{M}+\frac{\left(1-\exp \left(-x^{2}\right)\right)}{\left(1+\exp \left(-x^{2}\right)\right)} ; \quad \text { with } M \frac{\left(1-\exp \left(-x^{2}\right)\right)}{x^{2}\left(1+\exp \left(-x^{2}\right)\right)}
$$

whose energy levels are reflected in Fig. 8. Apart from this we also consider similar cases as 


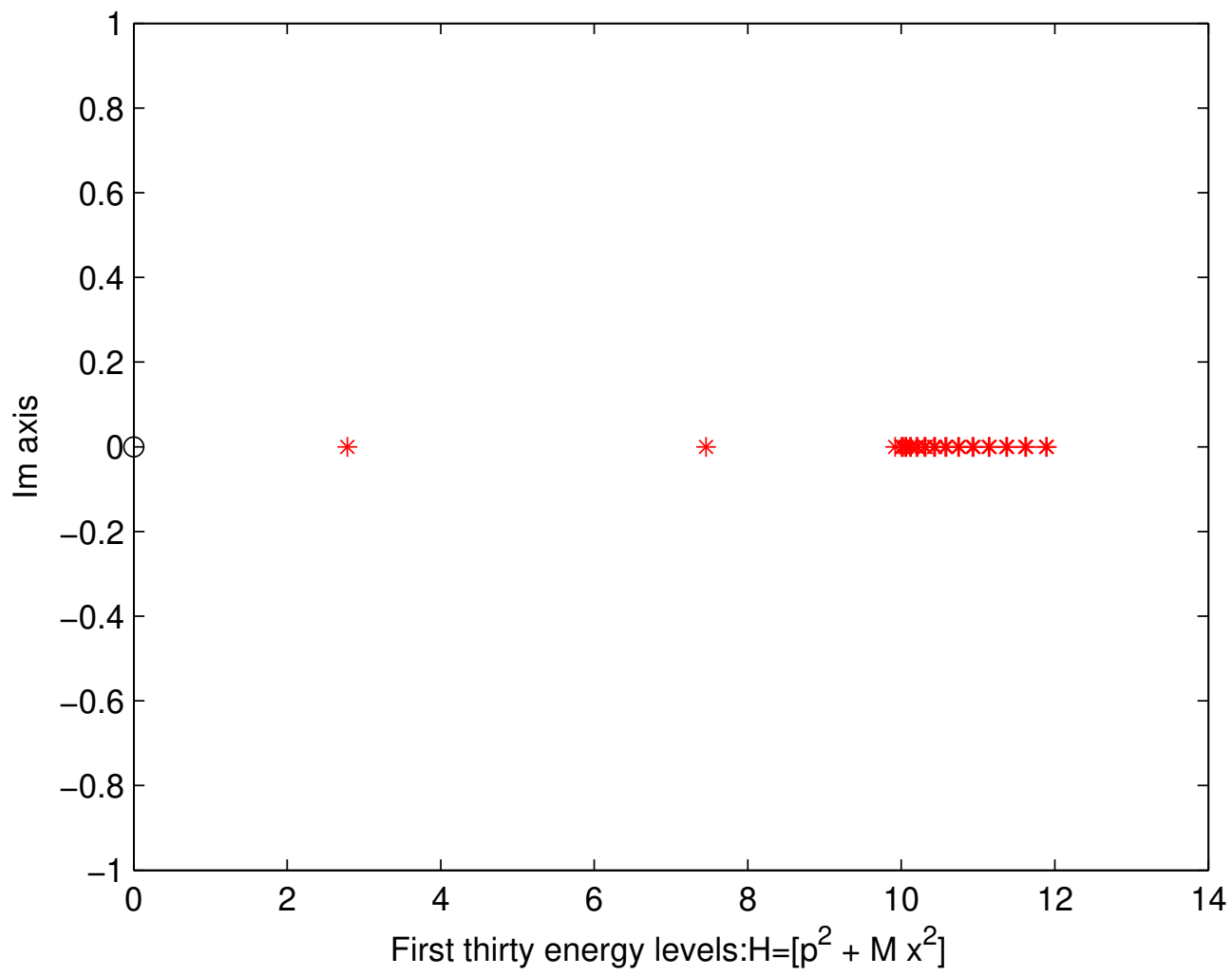

Figure 3: Energy levels of $H=p^{2}+M x^{2} ; \quad$ with $M=10 \frac{\left(1-\exp \left(-x^{2}\right)\right.}{x^{2}}$

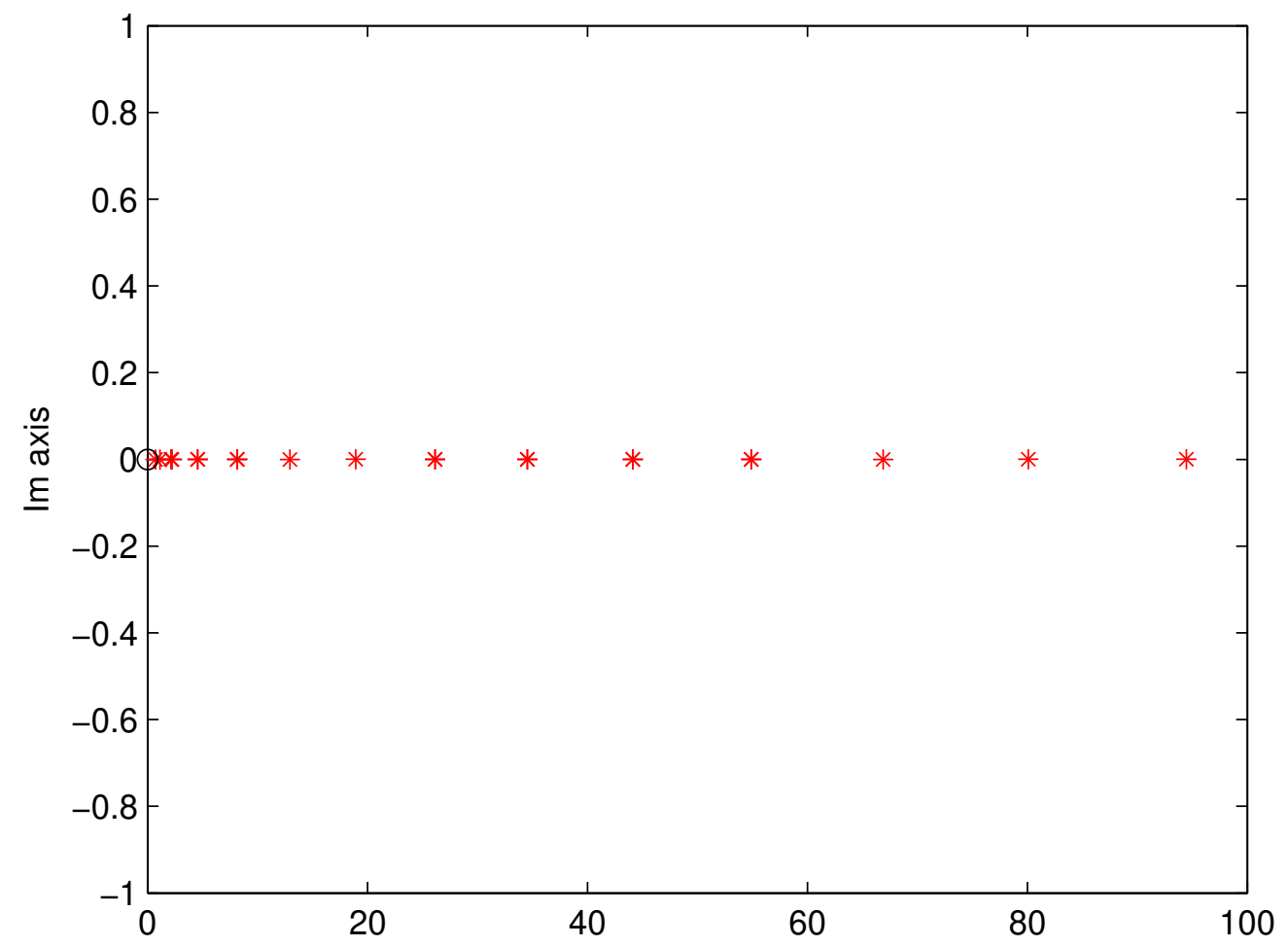

Real axis:Spectra of $\mathrm{H}=\mathrm{p}^{2} / \mathrm{M}+\left(1-\exp \left(-\mathrm{x}^{2}\right)\right) /\left(1+\exp \left(-\mathrm{x}^{2}\right) ; \mathrm{M}=\left(1-\exp \left(-\mathrm{x}^{2}\right)\right) / \mathrm{x}^{2}\left(1+\exp \left(-\mathrm{x}^{2}\right)\right.\right.$

Figure 8: Energy levels of $H=p^{2} / M+M x^{2} ; \quad M=\frac{\left(1-\exp \left(-x^{2}\right)\right.}{x^{2}\left(1+\exp -x^{2}\right)}$ 


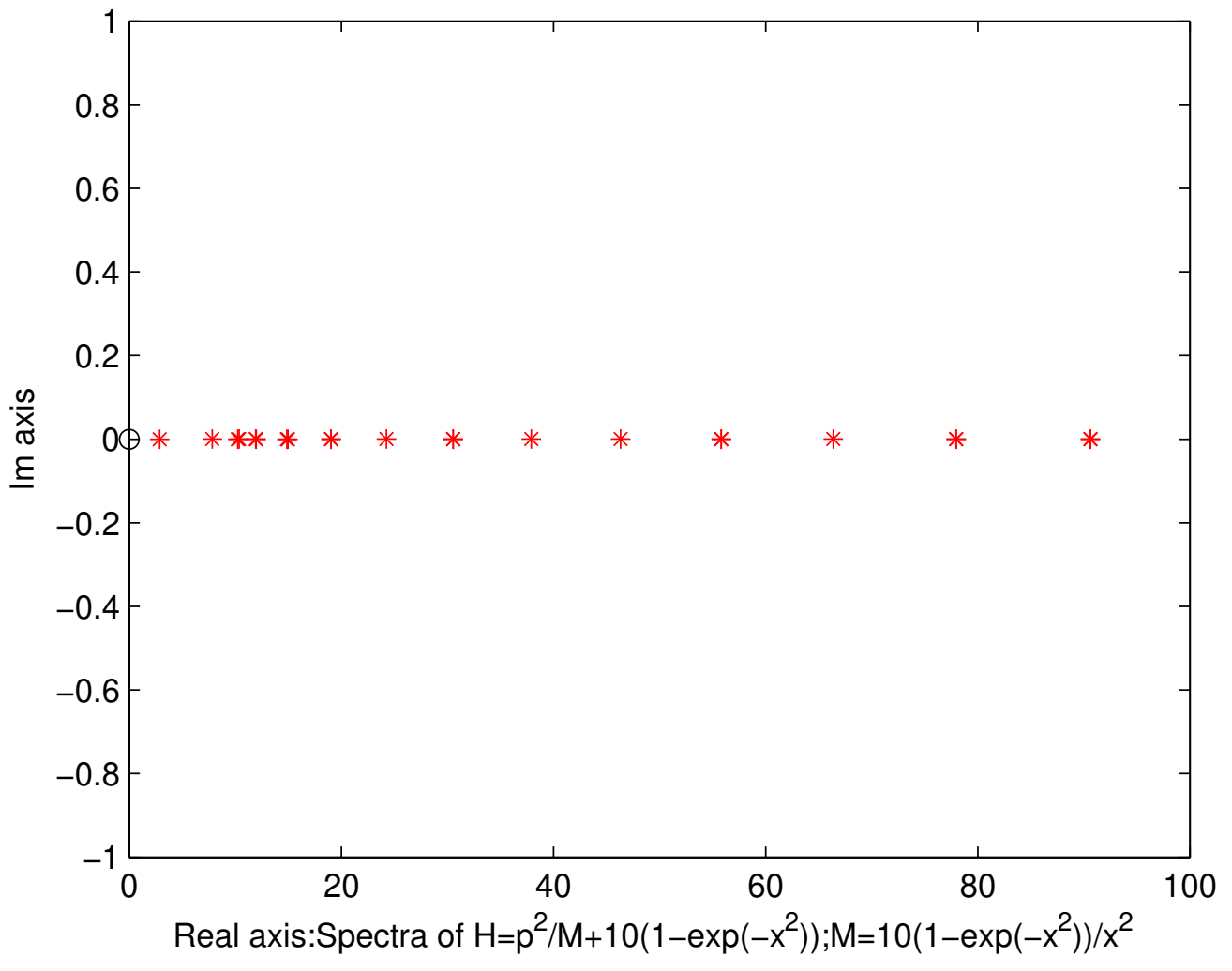

Figure 4: Energy levels of $H=p^{2} / M+M x^{2} ; \quad$ with $M=10 \frac{\left(1-\exp \left(-x^{2}\right)\right.}{x^{2}}$

$$
H^{(9)}=\frac{p^{2}}{M}+\frac{\left(1-\exp \left(-x^{2}\right)\right)}{\left(1+\exp \left(-x^{2}\right)\right)} ; \quad \text { with } M \frac{\left(1-\exp \left(-x^{2}\right)\right)}{x^{4}\left(1+\exp \left(-x^{2}\right)\right)}
$$

and

$$
H^{(10)}=\frac{p^{2}}{M}+\frac{\left(1-\exp \left(-x^{2}\right)\right)}{\left(1+\exp \left(-x^{2}\right)\right)} ; \quad \text { with } M \frac{\left(1-\exp \left(-x^{2}\right)\right)}{x^{6}\left(1+\exp \left(-x^{2}\right)\right)}
$$

Here, the energy levels are reflected in Figs. 9,10, respectively. These models are nontrivial because of the potential nature. The finite levels are due to the validity of condition $V(x) \ll 1$. 


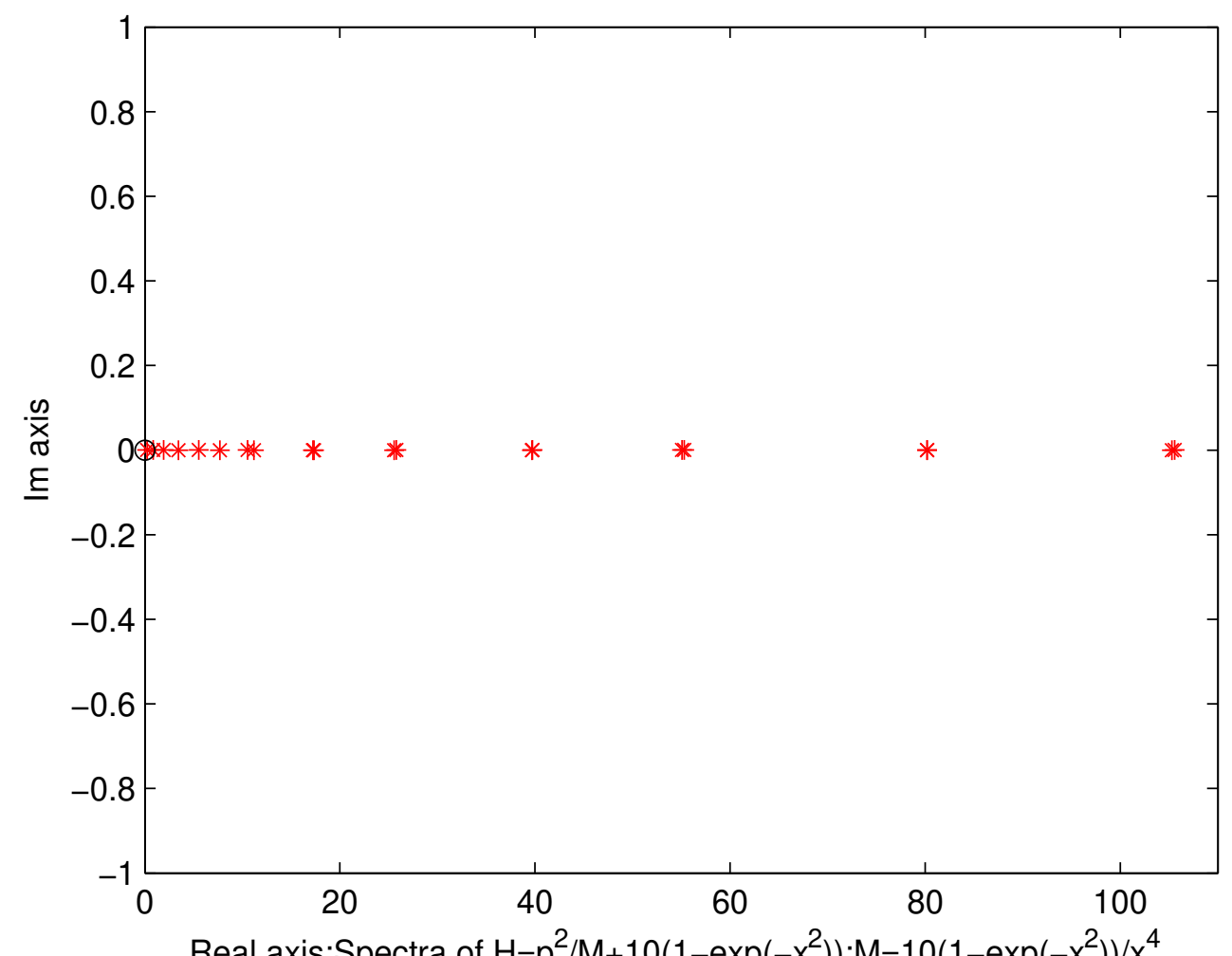

Figure 5: Energy levels of $H=p^{2} / M+M x^{4} ; \quad$ with $M=10 \frac{\left(1-\exp \left(-x^{2}\right)\right.}{x^{4}}$

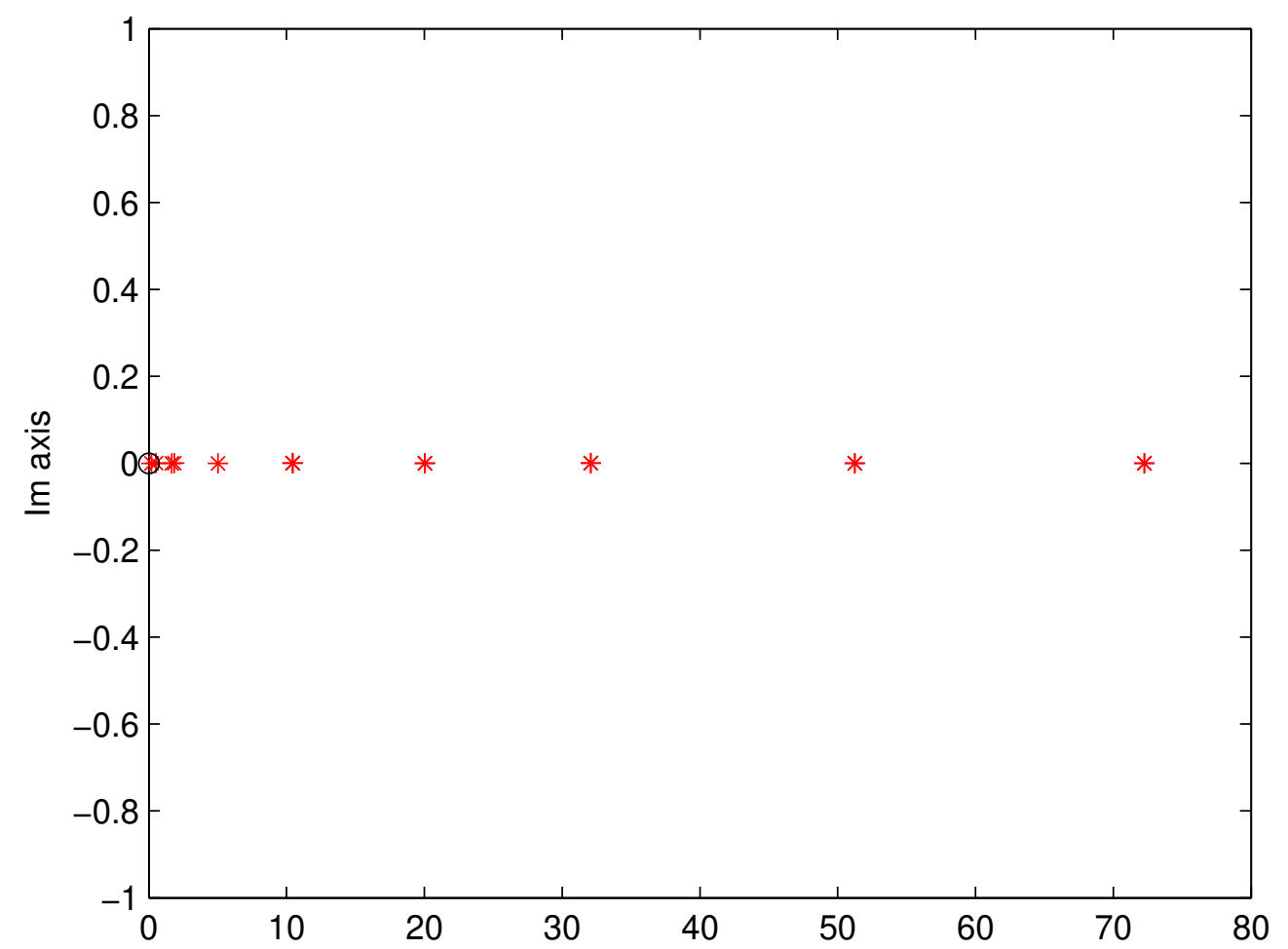

Real axis:Spectra of $\mathrm{H}=\mathrm{p}^{2} / \mathrm{M}+\left(1-\exp \left(-\mathrm{x}^{2}\right)\right) /\left(1+\exp \left(-\mathrm{x}^{2}\right) ; \mathrm{M}=\left(1-\exp \left(-\mathrm{x}^{2}\right)\right) / \mathrm{x}^{4}\left(1+\exp \left(-\mathrm{x}^{2}\right)\right.\right.$

Figure 9: Energy levels of $H=p^{2} / M+M x^{4} ; \quad M=\frac{\left(1-\exp \left(-x^{2}\right)\right.}{x^{4}\left(1+\exp -x^{2}\right)}$ 


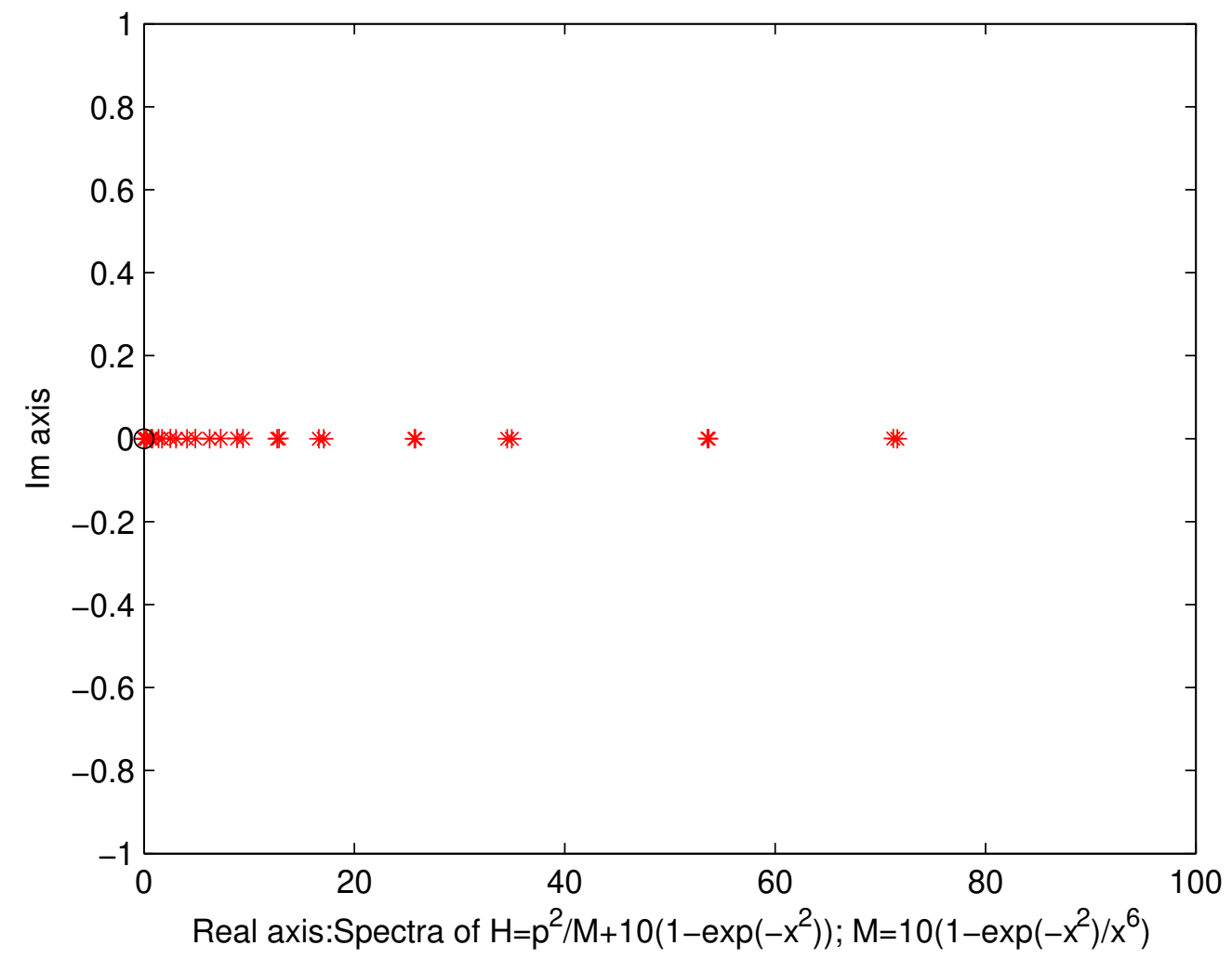

Figure 6: Energy levels of $H=p^{2} / M+M x^{6} ; \quad$ with $M=10 \frac{\left(1-\exp \left(-x^{2}\right)\right.}{x^{6}}$

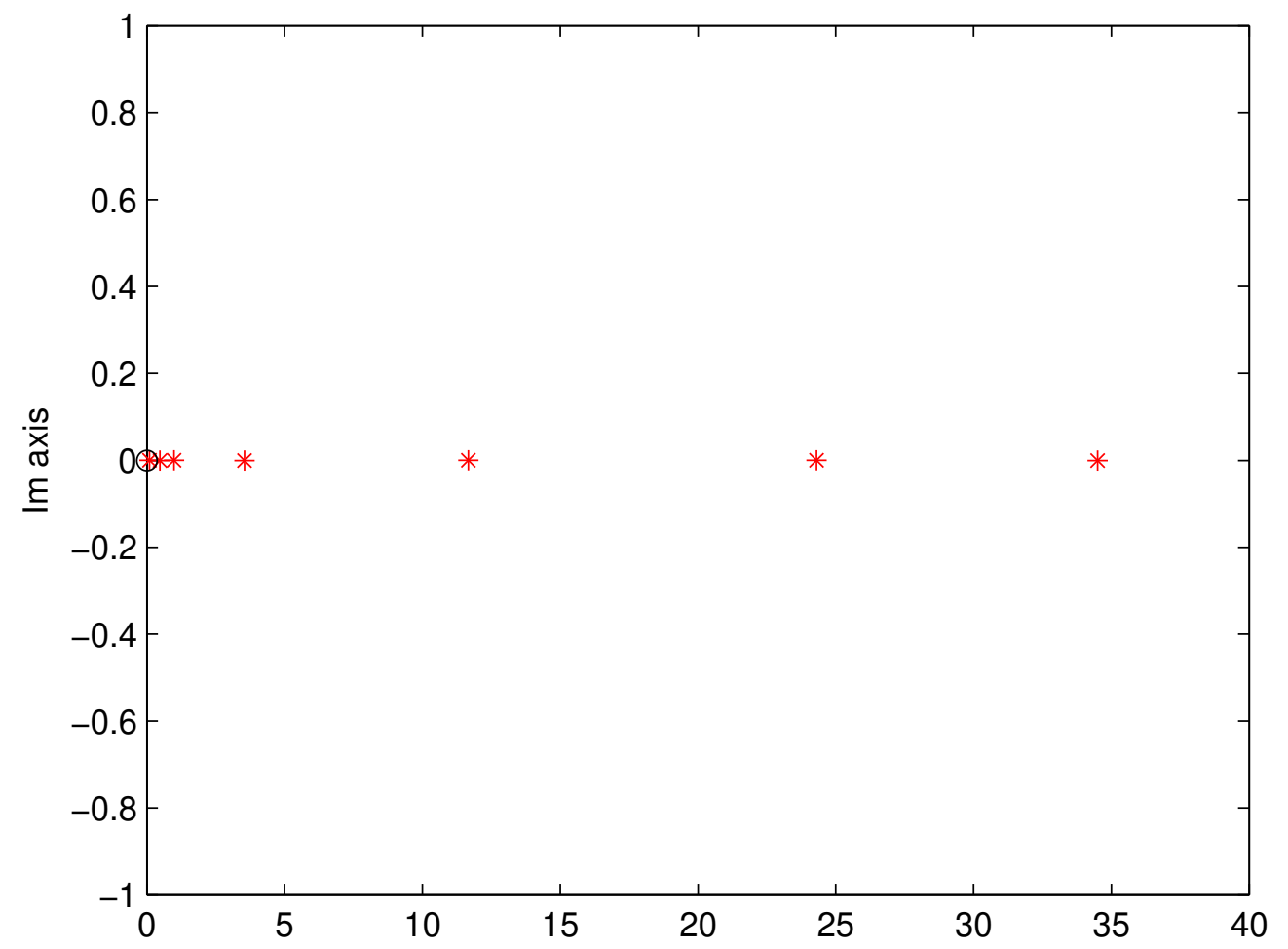

Real axis:Spectra of $\mathrm{H}=\mathrm{p}^{2} / \mathrm{M}+\left(1-\exp \left(-\mathrm{x}^{2}\right)\right) /\left(1+\exp \left(-\mathrm{x}^{2}\right) ; \mathrm{M}=\left(1-\exp \left(-\mathrm{x}^{2}\right)\right) / \mathrm{x}^{6}\left(1+\exp \left(-\mathrm{x}^{2}\right)\right.\right.$

Figure 10: Energy levels of $H=p^{2} / M+M x^{6} ; \quad M=\frac{\left(1-\exp \left(-x^{2}\right)\right.}{x^{6}\left(1+\exp -x^{2}\right)}$ 


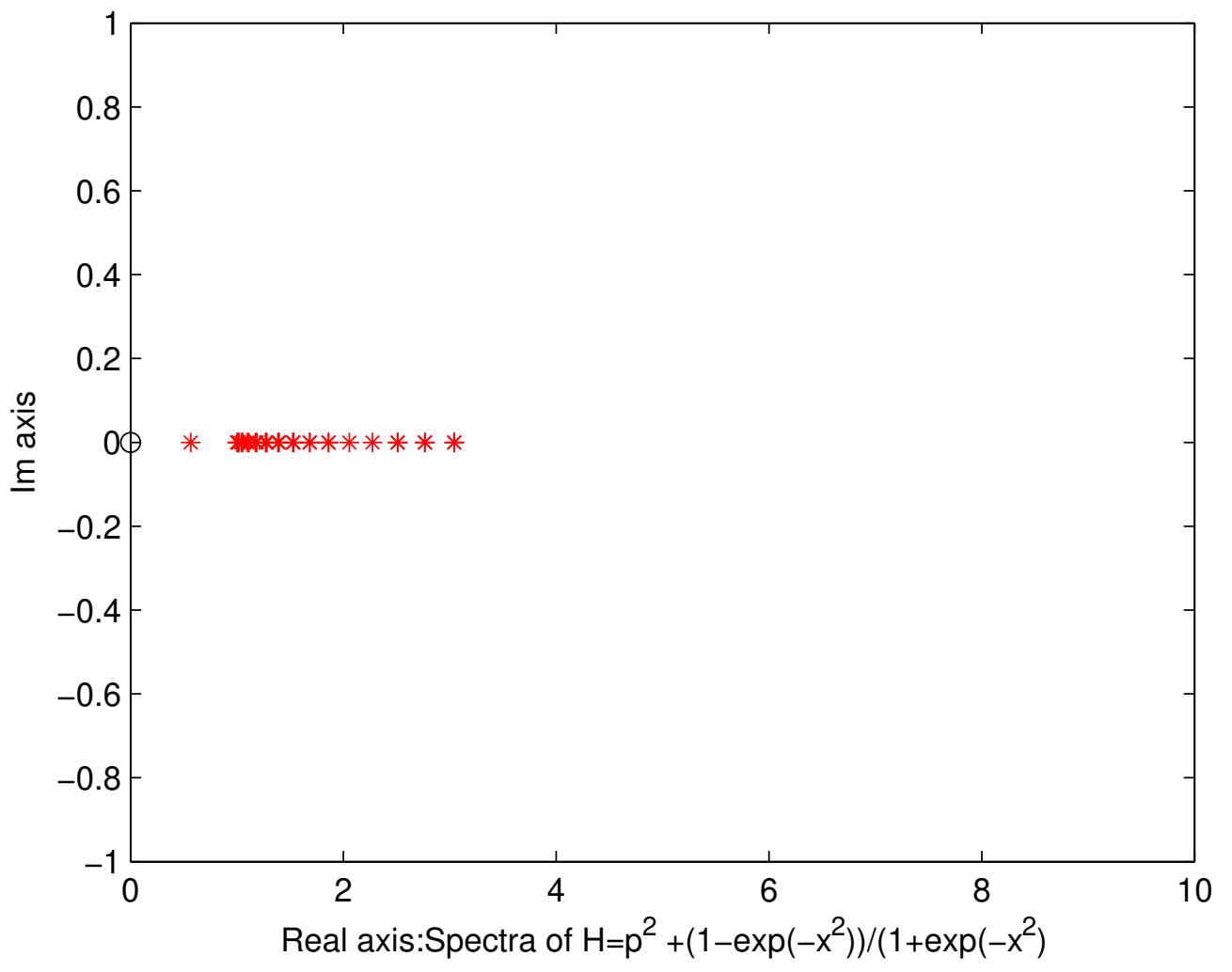

Figure 7: Energy levels of $H=p^{2}+\frac{\left(1-\exp -x^{2}\right)}{\left(1+\exp -x^{2}\right)}$

\section{Method of calculation}

In order to calculate the energy levels of above Hamiltonians, we use matrix diagonalisation method $[10,12]$ to mirror convergent energy levels, on solving the eigenvalue relation

$$
H|\Psi>=E| \Psi>
$$

with

$$
\left|\Psi>=\sum_{m} A_{m}\right| m>
$$

where $\mid m>$ satisfies the relation

$$
\left[H_{0}=p^{2}+x^{2}\right]|m>=(2 m+1)| m>
$$

\section{Conclusion}

In this paper we have shown that DDL quantum systems can reflect infinite distinct discrete energy levels under suitable form of position dependent mass. The model mass 
Table 1 : Fractional Harmonic Oscillator and energy levels comparison

\begin{tabular}{cccc}
\hline $\mathrm{n}$ & $\mathrm{g}$ & Present & Previous [6] \\
\hline 0 & 0.1 & 1.3805318 & 1.38053 \\
1 & & 4.0798830 & 4.0798 \\
2 & & 6.6679191 & 6.667 \\
3 & & 9.1665674 & \\
\hline 0 & 1 & 1.2323507 & 1.23235 \\
1 & & 3.5073883 & 3.50738 \\
2 & & 5.5897789 & 5.58977 \\
& & 7.6482012 & \\
\hline
\end{tabular}

was actually proposed by Cruz et al. [15] and used by others [1, 3, 7, 9]. It is worth mentioning that only this typical form of $\mathrm{T}$ can be derived [8]. No other form of von Roos model Hamiltonian $[13,14]$ has that advantage. In other words, all other forms can only be based on approximation [5]. This universality feature has been exploited in the above model Hamiltonians. All figures have plotted from the respective numerical calculation of energy levels. It should be borne in mind that all the figures on PDM actually we have plotted with T i.e $\frac{p^{2}}{M}=T$. In order to convince the reader about our method $[1,3]$, we consider two simple models as

$$
H_{\text {Mitra }}^{(1)}=p^{2}+x^{2}+\frac{x^{2}}{\left(1+0.1 x^{2}\right)}
$$

and

$$
H_{\text {Mitra }}^{(2)}=p^{2}+x^{2}+\frac{x^{2}}{\left(1+x^{2}\right)}
$$

and compare the present result with that of earlier computation by Mitra [6] in Table 1 .

Summarizing the above, we conclude that FDL( Finite Discrete Levels) $\Longrightarrow$ IDLPDM(Infinite Discrete Levels in Position Dependent Mass ) in quantum operators.

\section{Acknowledgements}

The authors Rabab Jarrar, Hussein Shanak, Jihad Asad would like to thank Palestine Technical university- Kadoorie for funding this work. Scientific research funding 2022.

\section{References}

[1] BG da Costa, IS Gomez, and M Portesi. $\kappa$-deformed quantum and classical mechanics for a system with position-dependent effective mass. Journal of Mathematical Physics, 61(8):082105, 2020.

[2] HF Jones. Comment on solvable model of bound states in the continuum (bic) in on dimension (2019, 94, 105214). Physica Scripta, 96(8):087001, 2021. 
[3] S Karthiga, V Chithiika Ruby, M Senthilvelan, and M Lakshmanan. Quantum solvability of a general ordered position dependent mass system: Mathews-lakshmanan oscillator. Journal of Mathematical Physics, 58(10):102110, 2017.

[4] PM Mathews and M Lakshmanan. On a unique nonlinear oscillator. Quarterly of Applied Mathematics, 32(2):215-218, 1974.

[5] PM Mathews and M Lakshmanan. A quantum-mechanically solvable nonpolynomial lagrangian with velocity-dependent interaction. Il Nuovo Cimento A (1965-1970), 26(3):299-316, 1975.

[6] AK Mitra. On the interaction of the type $\lambda \times 2 /(1+\mathrm{gx} 2)$. Journal of Mathematical Physics, 19(10):2018-2022, 1978.

[7] O Mustafa. Pdm creation and annihilation operators of the harmonic oscillators and the emergence of an alternative pdm-hamiltonian. Physics Letters A, 384(13):126265, 2020 .

[8] B Rath. Unpublished research work (communicated).

[9] B Rath. Comment on" pdm creation and annihilation operators of the harmonic oscillators and the emergence of an alternative pdm-hamiltonian" by omar mustafa [phys. lett. a 384 (2020) 126265]. Physics Letters A, 393:127174, 2021.

[10] B Rath. Real spectra in some negative potentials: linear and nonlinear onedimensional pt-invariant quantum systems. The European Physical Journal Plus, 136(5):1-10, 2021.

[11] B Rath and MKA Kaabar. A method on generation of finite level (s) in real and complex systems. Mathematics and Computational Sciences, 2(1):11-23, 2021.

[12] B Rath and HA Mavromatis. Energy-level calculation through modified hill determinant approach: for general oscillator. Indian Journal of Physics, 1999.

[13] O von Roos. Position-dependent effective masses in semiconductor theory. Physical Review B, 27(12):7547, 1983.

[14] O von Roos and H Mavromatis. Position-dependent effective masses in semiconductor theory. ii. Physical Review B, 31(4):2294, 1985.

[15] S Cruz y Cruz, J Negro, and LM Nieto. Classical and quantum position-dependent mass harmonic oscillators. Physics Letters A, 369(5-6):400-406, 2007. 